\title{
Fabrication of Porous Titania Particles from Complex Fluids by Spray Drying Process and Their Applications
}

\author{
Young-Sang Cho* \\ Department of Chemical Engineering and Biotechnology, Korea Polytechnic University, Siheung 15073, \\ Republic of Korea
}

\begin{abstract}
In this study, a spray drying system was used to prepare macroporous titania micro-particles for applications including UV blocking materials and photocatalysts. To synthesize the macroporous particles a colloidal templating method using polymeric beads as sacrificial templates and a titania nano-colloid as precursor material was adopted. The resulting porous titania particles were mixed with distilled water to measure their light transmittance, and to assess their UV blocking performance. To demonstrate another application, the organic dye Rhodamine B was decomposed using the macroporous titania micro-particles as a photocatalyst for water purification. The spray drying system was also utilized to fabricate porous iron oxide particles using iron chloride liquid precursor. Following a reduction reaction, the porous iron oxide particles fabricated in this study are also potentially applicable as intermediates for the synthesis of porous metal particles, which have various applications including porous surface burners, artificial bones, thermal insulation, and sound absorbers.
\end{abstract}

(Received June 28, 2016; Accepted September 21, 2016)

Keywords: porous titania particles, spray dying, UV blocking property, photocatalyst, porous iron oxide particles.

\section{INTRODUCTION}

Recently, extensive effort has been expended to synthesize porous materials for a wide range of applications, including catalytic supports, photonic pigments, as materials for sensors and electrodes, and as reinforcing composites [1-5]. In order to form architectures with high porosity and large surface area, various fabrication schemes have been developed, including hydrothermal synthesis, soft and hard templating methods, the rapid evaporation of droplets, electrospinning, and electrochemical anodization [6-11]. Among these various synthesis schemes, the colloidal templating method is considered a promising approach for forming ordered arrays of uniform pores due to its economic and facile nature [12].

Specifically, porous particulate materials have been synthesized using droplets as confining geometries, to encapsulate precursor materials and organic templates $[13,14]$. Aerosols or emulsions have been adopted as shrinking droplets for this purpose, to induce the

* Corresponding Author: Young-Sang Cho

[Tel: +82-31-8041-0612, E-mail: yscho78@kpu.ac.kr]

Copyright (c) The Korean Institute of Metals and Materials self-organization and formation of organic-inorganic hybrid particles. Subsequent heat treatment leads to the final porous particles. Among droplet-based synthesis protocols, these two steps of self-assembly and calcination have also been combined in a single apparatus, such as a spray pyrolysis reactor, allowing various macroporous particles to be fabricated within short time intervals $[15,16]$. However, during high-temperature operation of the pyrolysis reactor, the produced particles can become deposited on the inner wall of the furnace, which decreases the yield of the fabrication process. This has stimulated efforts to develop a more economic synthesis method that can eliminate the loss of the produced powder materials, using other preparation routes, such as spray drying apparatus.

The spray drying technique is currently used to fabricate various powder materials in the medical and food industries. As one successful example, Pulmosphere ${ }^{\mathrm{TM}}$, a porous powder which can be efficiently absorbed into human lungs, has been synthesized in this manner [17]. Various operating conditions, including drying temperature, can be adjusted during the spray drying process within a wide controllable range, which 
can promote the rapid evaporation of the droplets. By adjusting the drying conditions of the spray drying process, the morphologies of the final products can be easily controlled due to the change in mass Peclet number [18]. The powder collection conditions can also be changed by adjusting the feed rate of the hot air stream, thus minimizing powder loss.

Our previous research on the synthesis of porous particles using emulsion droplets as confining geometries enables the facile fabrication of porous silica particles using commercial silica nano-colloids as precursor materials [19]. A similar approach for synthesizing meso-macroporous particles could be applied to the fabrication of porous silica particles, using metal alkoxides as the source materials [20]. However, these methods have some drawbacks, most notably high cost, which is inevitable due to the continuous oil phase required during the emulsification step.

In addition, the fabrication of porous particle materials has mainly been limited to silica, since it has proven difficult to assemble various other metal oxides as porous structures using our previous emulsion-assisted self-assembly strategy. In contrast, the spray drying technique does not require a continuous phase liquid state, since the tiny droplets are generated as aerosols. Consequently, the spray drying approach, which is a method of self-organizing precursor materials inside droplets, can be a more economical way of fabricating porous particles.

A complex fluid is a broadly defined concept which includes colloidal suspensions, aerosols, foams, or emulsions. Complex fluids are primarily uniform mixtures of binary phases, and most can exhibit both liquid and solid-like behaviors under the external physical change. Colloidal suspensions of latex beads or inorganic particles, as well as solutions of polymers or micelles, are categorized as complex fluids [21].

In this study, the droplets of a complex fluid system, a hetero-colloidal dispersion, were studied to demonstrate the formation of porous particles by evaporation-induced self-assembly. Specifically, macroporous titania micro-particles were prepared by the spray drying process, using a titania nano-colloid and polystyrene nanospheres as the precursor and templating materials, respectively. Self-organization of the mixed dispersion of titania nanoparticles and polymeric beads was induced inside the drying chamber of the spray drying system to obtain organic-inorganic composite particles. After collecting the composite powder from the collection chamber, calcination was performed to remove the organic templates, and to fabricate the final porous titania micro-particles. To demonstrate an application of the fabricated macroporous titania micro-particles, their feasibility as UV blocking materials and photocatalysts was studied. A similar selfassembly strategy was adopted to synthesize porous iron oxide micro-particles using an aqueous iron chloride solution as the precursor material.

\section{EXPERIMENTAL}

\subsection{Materials}

To synthesize the macroporous titania particles, a commercial titania nano-colloid obtained from Nanoamor Inc was used as the precursor material. To synthesize the polystyrene nanospheres, styrene monomer (99\%) and the initiator, and potassium peroxodisulfate (KPS, 99\%) were purchased from Daejung Chemicals and Kanto Chemicals, respectively. Acrylic acid (99.5\%) was used as the comonomer, bought from Samchun Chemicals. To test the photocatalytic performance of the porous titania particles, Rhodamine B purchased from Junsei Chemicals was used as the organic dye. For the fabrication of the porous iron oxide particles, iron (III) chloride hexahydrate $\left(\mathrm{FeCl}_{3} \cdot 6 \mathrm{H}_{2} \mathrm{O}\right)$ was purchased from Samchun chemicals.

\subsection{The synthesis of the monodisperse polystyrene nanospheres}

$43 \mathrm{ml}$ styrene was added to a batch-type emulsion polymerization reactor containing $380 \mathrm{ml}$ distilled water under vigorous stirring at $300 \mathrm{rpm}$. The temperature of the reactor was maintained at $70{ }^{\circ} \mathrm{C}$. Then, the proper amount of acrylic acid was added to the reactor. To start the polymerization reaction, $0.196 \mathrm{~g}$ of KPS dissolved in 13.75 $\mathrm{ml}$ distilled water was added to the reactor after nitrogen 
Table 1. The synthesis conditions of the polystyrene nanospheres with uniform diameter.

\begin{tabular}{c|c|c|c|c}
\hline Chemicals & Water & Styrene & Acrylic acid & KPS \\
\hline $\begin{array}{c}\text { Amount } \\
\text { Used }\end{array}$ & $393.75 \mathrm{ml}$ & $43 \mathrm{ml}$ & $0.7875 \mathrm{~g}$ & $0.196 \mathrm{~g}$ \\
\hline
\end{tabular}
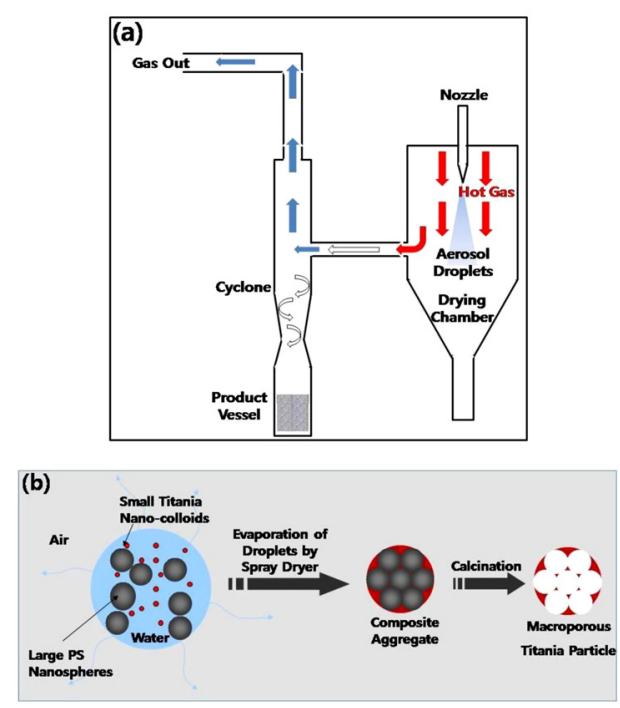

Fig. 1. (a) Schematic figure of spray drying apparatus and (b) the fabrication process of macroporous titania micro-particles by spray drying technology.

purging for at least 1 hour. The polymerization was continued for 18 hours. The detailed synthesis conditions are summarized in Table 1. After completion of the reaction, the particulate products were filtered and the volatile monomers remaining in the suspension were evaporated under a chemical hood at room temperature for a few days.

\subsection{Synthesis of the macroporous titania micro- particles}

A $100 \mathrm{ml}$ suspension of polystyrene nanospheres (5 wt\%) was mixed with $7 \mathrm{ml}$ commercial titania nano-colloid (36 $\mathrm{wt} \%)$ and $53.5 \mathrm{ml}$ distilled water to prepare the precursor solution for the spray drying process. The hetero-colloid was fed through two fluid nozzle and dried at $200{ }^{\circ} \mathrm{C}$ inside the drying chamber. The resulting powder was collected from the collection section of the spray dryer, and further heat treatment was performed at $500{ }^{\circ} \mathrm{C}$ for 5 hours to produce the macroporous titania micro-particles.

\subsection{Photocatalytic decomposition of Rhodamine B} dye

The concentrations of the Rhodamine B dye and the porous titania particles were adjusted to $0.0005 \mathrm{~g} / \mathrm{L}$ and $0.002 \mathrm{~g} / \mathrm{L}$, respectively. After preparing an aqueous dispersion containing each component, external light was blocked and a dark condition was maintained for 30 minutes to reach equilibration. Then, UV light (F10T8 BLB, $10 \mathrm{~W}$, peak wavelength at 352 and $369 \mathrm{~nm}$, Sankyo Denki) was irradiated on the mixture to decompose the Rhodamine $\mathrm{B}$ and the concentration of remaining dye molecules was measured at regular time intervals to observe the reduction in dye concentration.

\subsection{Synthesis of porous iron oxide particles}

$3.5 \mathrm{ml}$ iron chloride hexahydrate $\left(\mathrm{FeCl}_{2} \cdot 6 \mathrm{H}_{2} \mathrm{O}\right)$ was mixed with a $25 \mathrm{ml}$ suspension of polystyrene nanospheres and 12 $\mathrm{ml}$ of distilled water to prepare a precursor solution for the spray drying process. To prepare porous iron oxide particles, the inlet hot air temperature was adjusted to $160{ }^{\circ} \mathrm{C}$ inside the drying chamber, and after spraying, a final calcination was conducted at $500{ }^{\circ} \mathrm{C}$ or $780{ }^{\circ} \mathrm{C}$ for 5 hours.

\subsection{Characterizations}

The morphologies of the macroporous particles were observed using a field emission scanning electron microscope (FE-SEM, Hitachi-S4700). The morphologies of the particles were also observed using transmission electron microscope (TEM, Tecnai G20, F20). The crystallinity of the porous titania powder was analyzed using the power X-ray diffraction technique (D/MAX-2200/PC). The UV-visible spectrum of the aqueous Rhodamine B solution was measured using the UV-visible spectrometer (Model: OPTIZEN POP).

\section{RESULTS AND DISCUSSION}

In this study, a spray drying process was adopted to induce the self-assembly of precursor materials, for the synthesis of porous particles. The structure of the spray drying system is schematically described in Fig. 1(a). In this method, a dispersion containing precursor materials is atomized after 

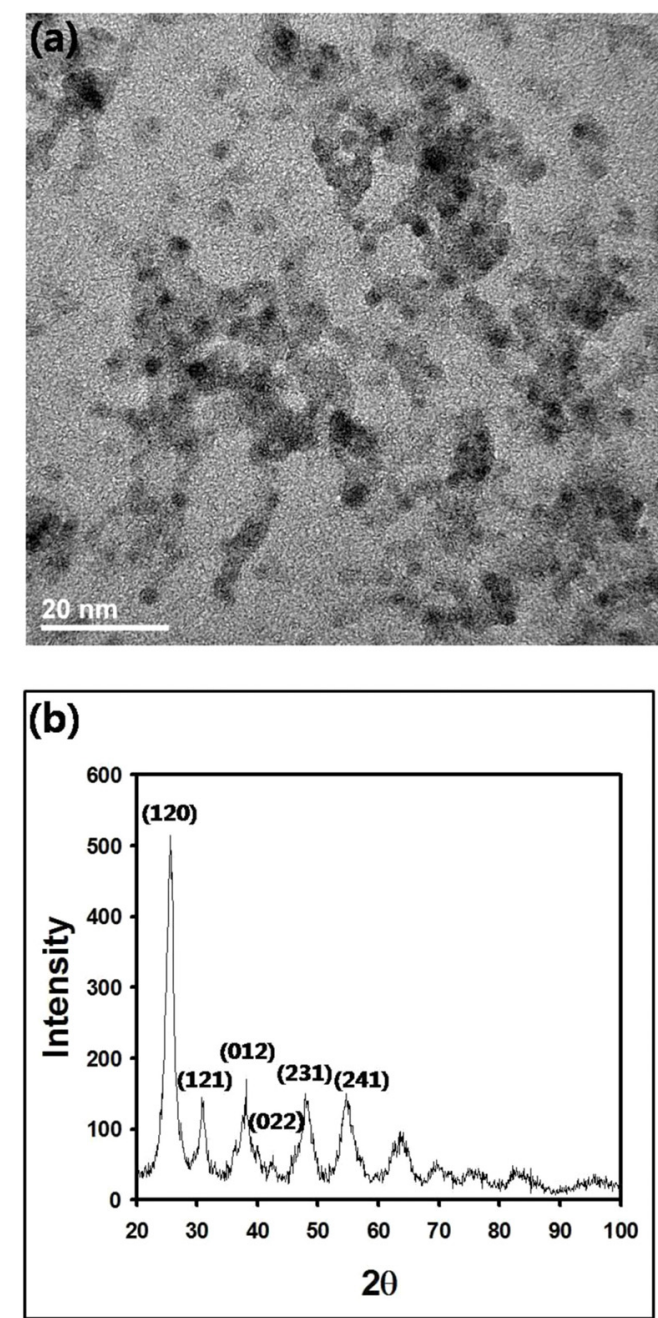

Fig. 2. (a) TEM image of commercial titania nano-colloid. Scale bar indicates $20 \mathrm{~nm}$. (b) Powder X-ray diffraction of the titania nano-colloid.

passing through two fluid nozzles which are typically used for the continuous generation of aerosol droplets. The droplets are injected into a drying chamber, where a supply of hot air is delivered to evaporate the droplets. The hot air inlet temperature can be adjusted by the operator to control the evaporation rate of the droplets. The vaporization of the droplets' dispersion medium induces an inward capillary pressure, resulting in shrinkage of the droplets and self-assembly of the materials dispersed in the droplets. Subsequently, a dried powder can be collected by cyclone in the collection section, for post-treatments such as calcination.

In this study, we fabricated macroporous titania micro-particles using this spray drying process. Figure $1 \mathrm{~b}$
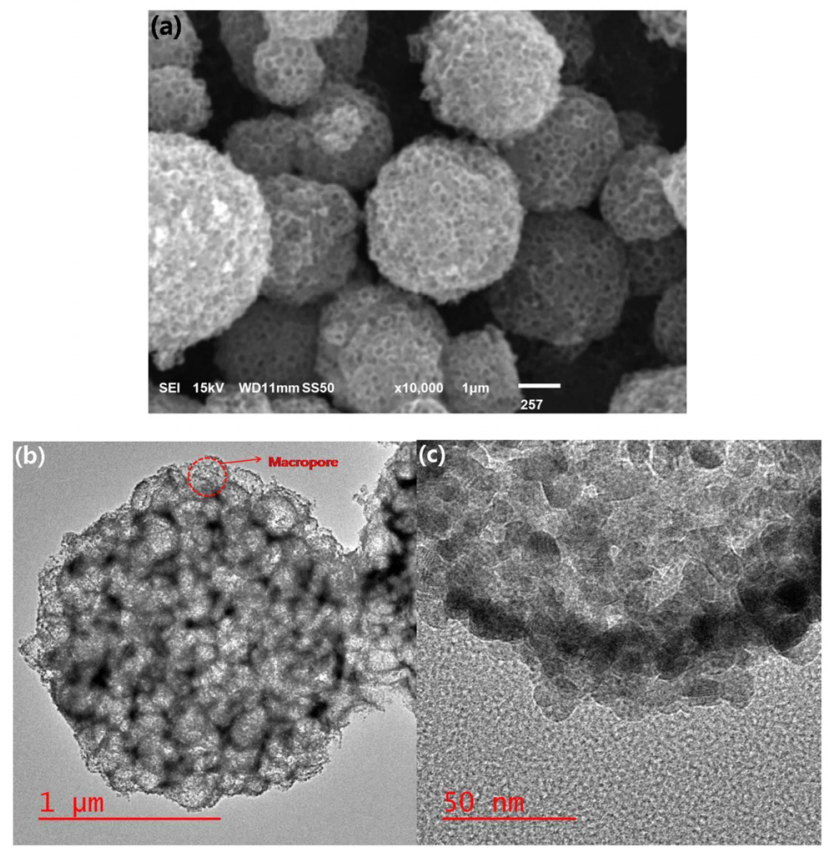

Fig. 3. (a) SEM and (b) TEM images of macroporous titania microparticles fabricated by spray drying process using inlet temperature of hot air as $200{ }^{\circ} \mathrm{C}$. Scale bars indicate $1 \mu \mathrm{m}$. (c) TEM image of the macroporous titania micro-particles. Scale bar is $50 \mathrm{~nm}$.

illustrates the mechanism by which the porous particles are formed using the spray drying process, which occurs by droplet-assisted self-organization. In the process, monodisperse polystyrene nanospheres were uniformly mixed with the titania nano-colloid, and then atomization of the hetero-colloidal dispersion was conducted using the two fluid nozzles of the spray drying system. The aerosol droplets containing the hetero-colloid of polymeric beads and titania nanoparticles were evaporated by applying hot air, to induce inward capillary pressure. Finally, the composite particles that were formed in the drying chamber were injected into the collection chamber. Subsequent calcination of the composite particles leads to macroporous titania micro-particles with high porosity and specific surface area, which can be applied to various industrial products such as cosmetics or photocatalysts.

Figure 2(a) shows a TEM image of the commercial titania nano-colloid which was used as the source material for fabricating the macroporous titania microparticles. The rod-like morphologies of the titania nanoparticles can be 

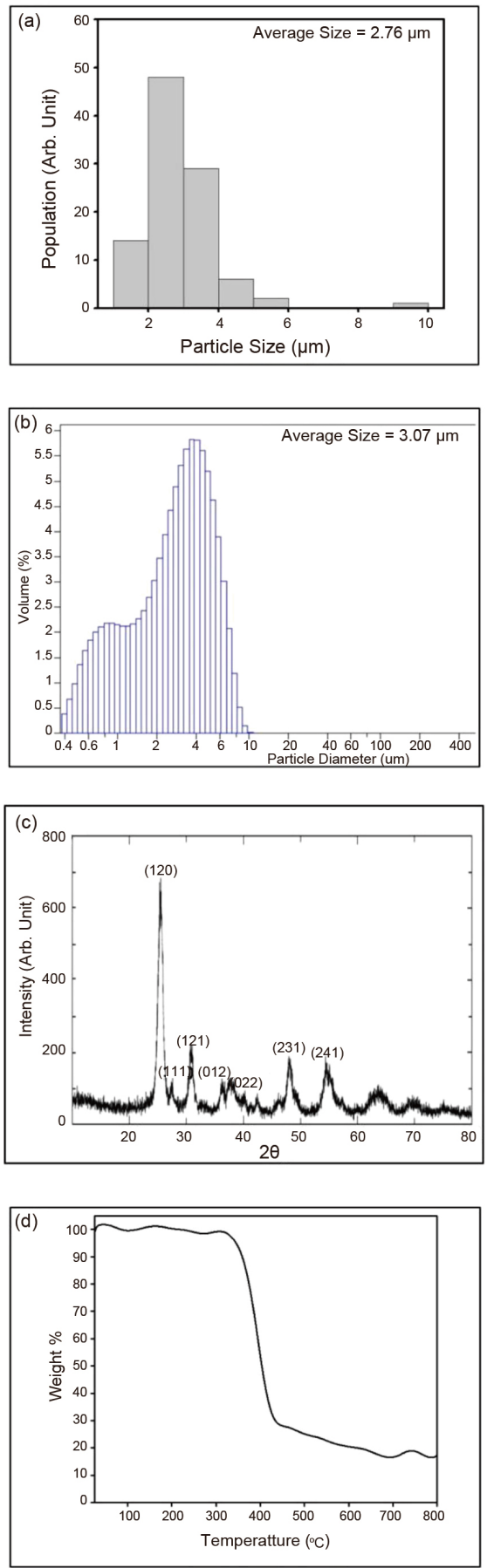

Fig. 4. Particle size distributions of macroporous titania particles measured by (a) electron microscope images in Fig. 3 and (b) particle size anlayzer. (c) Powder X-ray diffraction result of the porous titania particles. (d) TGA result of the particles.

observed, and the average particle size can be estimated to be a few nanometers. The crystallinity of the titania nanoparticles was confirmed by powder X-ray diffraction analysis, as shown in the graph in Fig. 2(b). From the diffraction pattern of the nanoparticles, a brookite structure was observed.

Figure 3(a) contains a SEM image of the macroporous titania micro-particles that were fabricated using the titania nano-colloid displayed in the TEM image in Fig. 2(a) as the titanium dioxide source material. Polystyrene nanospheres with $384 \mathrm{~nm}$ in diameter were used as templates, and were removed by calcination at $500{ }^{\circ} \mathrm{C}$ to form the macropores. The spray drying was conducted at a hot air inlet temperature of $200{ }^{\circ} \mathrm{C}$ inside the drying chamber. The inorganic nanoparticles and polymeric latex beads contained in the atomized droplets were self-assembled as the droplets were evaporated, and after calcination, macroporous titania particles were produced, as displayed in the SEM image in Fig. 3(a).

In addition to the SEM image, the porous structure of the titania micro-particles can also be confirmed from the TEM image in Fig. 3(b). A number of macropores with spherical shapes were formed by the removal of the latex beads, as indicated by the dotted line in the microscope image. A magnified TEM image of the porous titania particles was also obtained to observe the nano-structure of the particles, and is displayed in Fig. 3(c). It is evident that individual titania nanoparticles were assembled throughout the entire micro-structure of the porous particles after the self-organization induced by the spray dryer. The lattice structure of the titania nanoparticles can be also observed in the TEM image in Fig. 3(c), indicating that the original precursor material has a crystalline structure.

Figure $4 \mathrm{a}$ displays the particle size distribution of the macroporous titania micro-particles shown in the electron microscope images in Fig. 3. About a hundred particle diameters were measured to obtain the histogram contained in the graph in Fig. 4(a), and the size distribution was obtained using an average particle size of $2.76 \mu \mathrm{m}$. The size of the porous titania particles is micron-range, and consequently the particles are difficult to absorb through the skin, thereby decreasing the risk of possible skin toxicity when the porous particles are used as photocatalysts or as raw materials in cosmetics.

Figure 4(b) contains the size distribution of the porous 

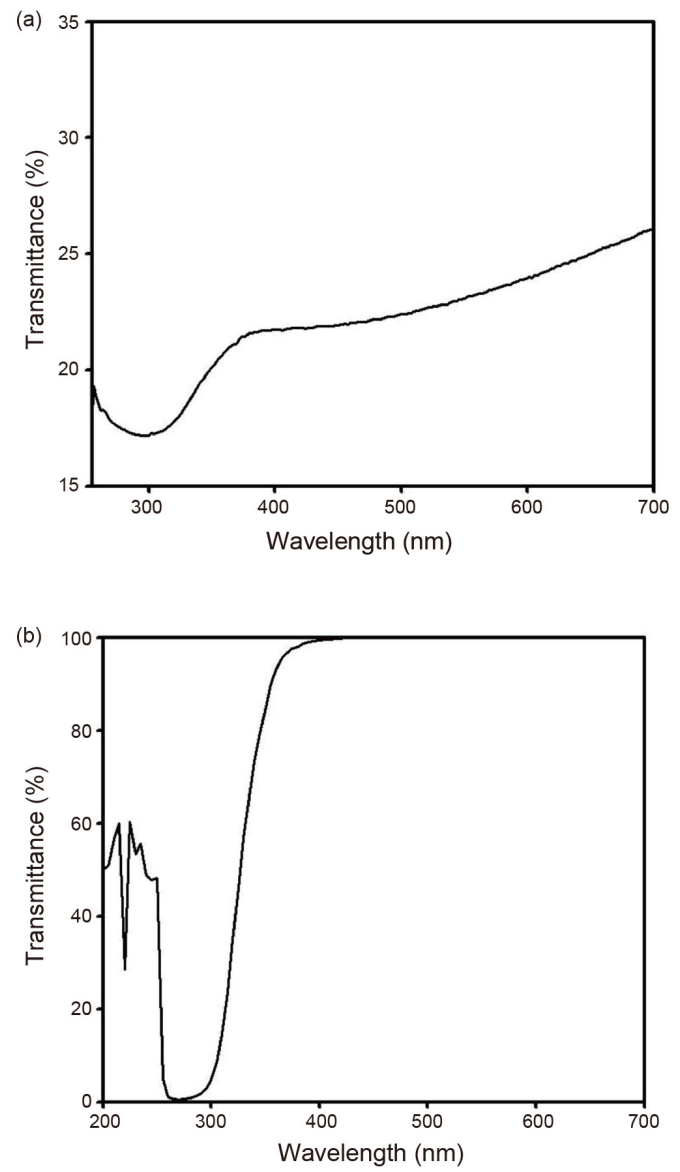

Fig. 5. UV-visible transmittance spectra of (a) macroporous titania micro-particles fabricated by spray drying technology and (b) commercial titnaia nano-colloids.

titania particles as measured by particle size analyzer. The average size was found to be $3.07 \mu \mathrm{m}$, which is slightly larger than the mean diameter estimated from the electron microscope image, and is possibly due to the aggregation of particles after calcination.

Figure 4(c) contains the powder X-ray diffraction pattern of the macroporous titania micro-particles synthesized by the spray drying process, showing that the crystalline structure of the porous particles is the brookite phase, which is the same as the crystal structure of the original titania nano-colloid used as the source material, shown in Fig. 2. Thus, the crystallinity of the titania nanoparticles was maintained during the drying of the droplets and subsequent calcination step, implying that the physicochemical properties of the source materials can be retained in the final macroporous titania microparticles. Figure 4(d) displays the TGA result of the particles fabricated by the spray drying process. From this graph, it can be concluded that, to form macropores by calcination, the organic templates should be removed at a temperature of at least $450{ }^{\circ} \mathrm{C}$.

Titania nanoparticles are currently being used as additive materials in the cosmetic industry, since these nano-materials have an intrinsic UV blocking property, making them suitable for sun cream products. However, due to their ultra-small sizes, the titania nanoparticles may result in skin toxicity. To prevent the powder materials from penetrating into human skin, micron-sized titania particles with comparable UV blocking properties are required.

In this context, the macroporous titania microparticles synthesized in this study can be utilized as UV blocking materials without skin toxicity, since the particle size is in the micron-range. The UV blocking property of the fabricated porous particles was confirmed by measuring the transmittance of the titania particles in UV and visible light wavelengths. As displayed in Fig. 5(a), a significant reduction in the transmittance spectrum was observed from 260 to 350 $\mathrm{nm}$, indicating that the macroporous titania micro-particles are suitable raw materials for this application in the cosmetics industry.

Nonetheless, the original commercial titania nanoparticles showed superior UV blocking performance compared to the fabricated macroporous titania micro-particles, as shown by the UV-visible spectrum in Fig. 5(b). The deterioration in the UV blocking performance of the porous titania micro-particles compared to the untreated titania nanoparticles can be attributed to a reduction in the intensity in UV light scattering as a result of the enlargement in particle size after self-assembly.

To demonstrate another application of our macroporous titania micro-particles, their photocatalytic reaction performance was tested for the decomposition of organic dyes such as Rhodamine B. Figure 6(a) shows the change in dye concentration as a function of UV irradiation time, showing that the Rhodamine $\mathrm{B}$ molecules were decomposed rapidly during the initial stage of the reaction period. When the commercial titania nano-colloid shown in the TEM image of Fig. 2(a) was also used as a photocatalyst for comparison, a 

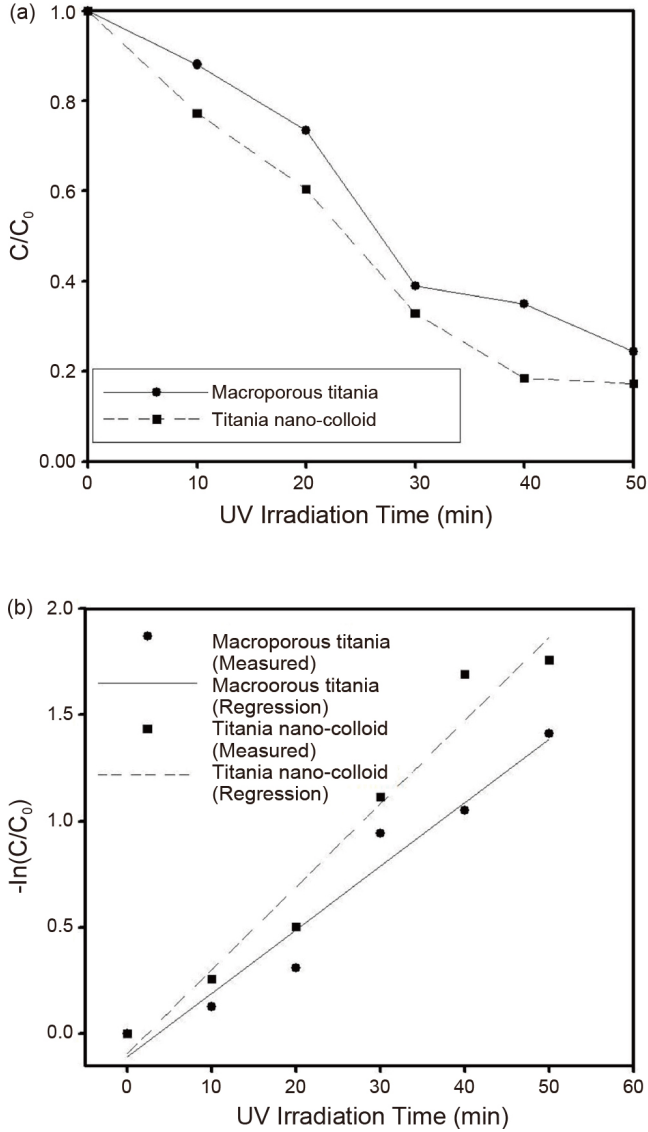

Fig. 6. (a) The concentration of Rhodamine B as a function of UV irradiation time showing the photocatalytic performance of the macroporous titania micro-particles and titania nano-colloid. (b) $\ln \left(\mathrm{C} / \mathrm{C}_{0}\right)$ as a function of UV irradiation time for the photocatalytic decomposition of Rhodamine B dyes.

relatively lower concentration of organic dye was measured during the decomposition reaction, compared to the results obtained using the macroporous titania micro-particles, as displayed in the graphs in Fig. 6(a).

The photocatalytic decomposition of organic dyes can be described using the following first order reaction, with an apparent rate constant $k_{a p p}[22]$.

$$
\frac{d C}{d t}=-k_{a p p} C
$$

To estimate the rate constants of the macroporous titania micro-particles and the commercial titania nano-colloid, the slope of the regression lines were calculated from the graphs shown in Fig. 6(b). The apparent rate constant, $k_{a p p}$ was
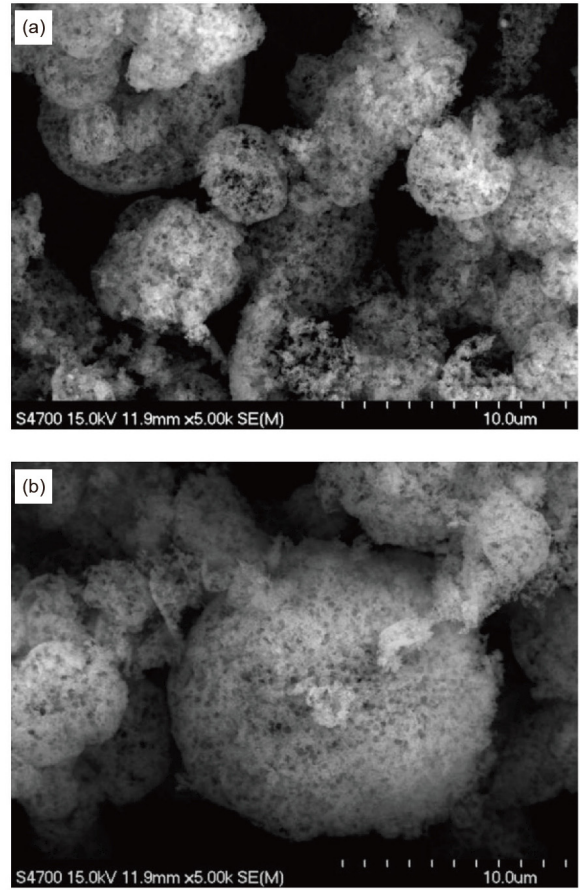

Fig. 7. (a, b) SEM image of macroporous titania particles fabricated with rutile titania nanoparticles.

calculated from the semi-log plot to be 0.0299 and 0.0391 $\min ^{-1}$ for the porous titania particles and the nano-colloid, respectively, as shown in Fig. 6(b). Thus, the commercial titania nano-colloid can be considered as a more efficient photocatalyst compared to the macroporous titania microparticles, which have a smaller rate constant.

However, the titania nano-colloid can be a more dangerous material due to its skin toxicity, which results from the ultra-small size of the nanoparticles, which are more easily absorbed than the macroporous titania micro-particles [23]. On balance, it can be concluded that the macroporous titania micro-particles synthesized by spray dryer might be the more attractive photocatalyst because of their suitable rate constant and reduced skin toxicity.

Figure 7 presents a SEM images of porous titania particles that were fabricated using a rutile titania nano-colloid, instead of brookite nanoparticles. The drying temperature was kept at $160{ }^{\circ} \mathrm{C}$ to induce the evaporation of the aerosols and self-assembly. Using the titania nano-colloid with a rutile phase as the starting material, spherical porous titania particles could be obtained, without anatase or brookite 


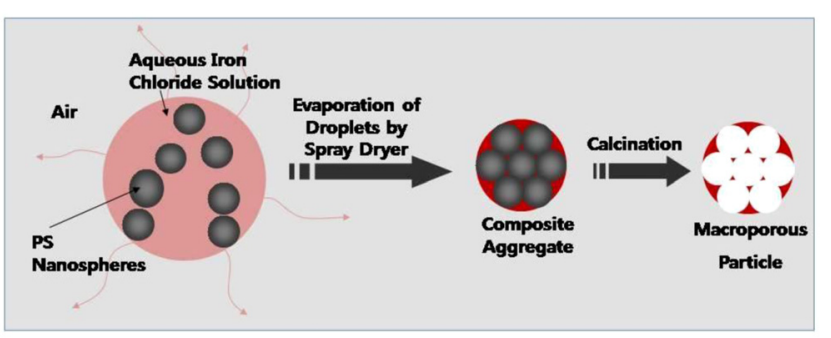

Fig. 8. Schematic figure for the fabrication process of porous iron oxide particles by droplet-assisted self-assembly strategy.

crystallinity.

In addition to the aerosol-assisted self-organization method described in this article, macroporous titania particles can be also produced using an emulsion-assisted self-assembly scheme, as reported in our previous articles [24,25]. Since the continuous phase of the droplets in the spray drying process is air, the aerosol-assisted method is more economical, because the raw material is much cheaper than that required by the process based on emulsification.

However, clogging of the two fluid nozzle needs to be prevented during the preparation of the feed solution in order to guarantee the hetero-colloidal solution is stably dispersed during the spray drying process. It should be noted that clogging was not observed when the dispersed phase was emulsified using a conventional emulsification apparatus, such as a homogenizer. To produce porous particles for various applications it is possible to choose the appropriate synthesis method by considering the advantages and disadvantages of the spray drying process.

Thus far, the fabrication and applications of macroporous titania micro-particles prepared using the spray drying process have been described in detail. To fabricate these porous titania particles, a nano-colloid was chosen as the precursor material for the self-assembly process. In addition to nanoparticles, liquid phase precursor materials can be also employed for the synthesis of porous powders. For instance, using an aqueous solution of iron chloride as the starting material, porous iron oxide particles can be obtained.

Figure 8 presents a schematic illustration the process for fabricating porous iron oxide particles using the selforganization of a complex fluid inside shrinking droplets.
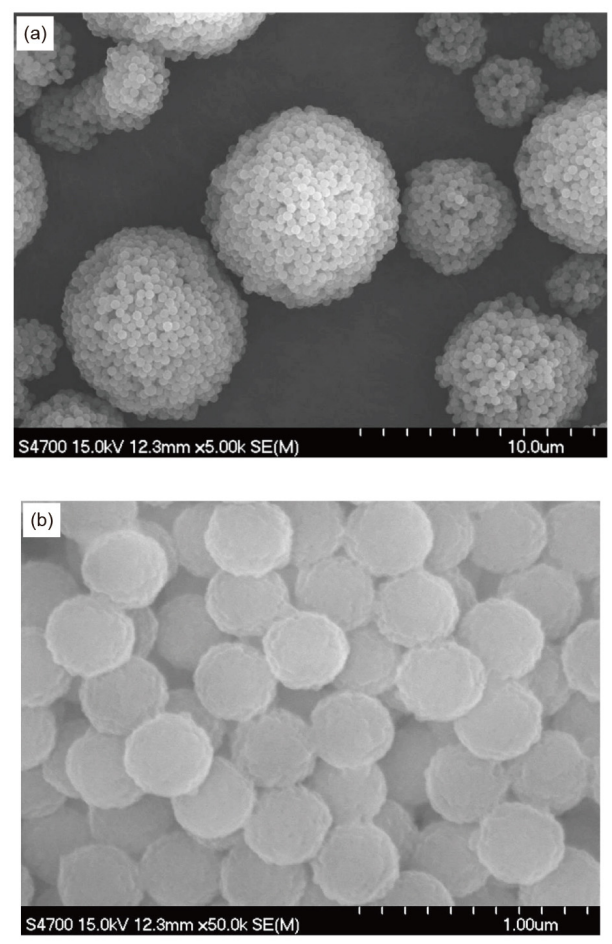

Fig. 9. (a, b) SEM images of composite particles of polystyrene nanospheres and iron chloride fabricated by spray drying process. The inlet temperature of hot air was controlled as $160{ }^{\circ} \mathrm{C}$. The scale bars indicate (a) 10 and (b) $1 \mu \mathrm{m}$, respectively.

Polymeric nanospheres with $464 \mathrm{~nm}$ in diameter and inorganic precursors such as metal chlorides are mixed uniformly in an aqueous dispersion medium. Using the two fluid nozzle in the spray dryer this complex fluid system can be atomized as tiny droplets containing the organic/inorganic components. By applying thermal energy, these droplets can be evaporated, resulting in shrinkage of the droplets, causing an inward capillary pressure. Self-organization can be induced by this compressive interfacial force and organic/inorganic composite particles can be fabricated. After suitable heat treatment, such as calcination, the polymeric nanospheres can be selectively removed, yielding porous particles as the final product.

Figure 9 shows SEM images of composite particles composed of polystyrene nanospheres and iron chloride, which were used as the organic template and inorganic precursor, respectively. The spherical morphologies of the composite particles can be observed in the microscope image shown in Fig. 9(a), implying that the original droplets 

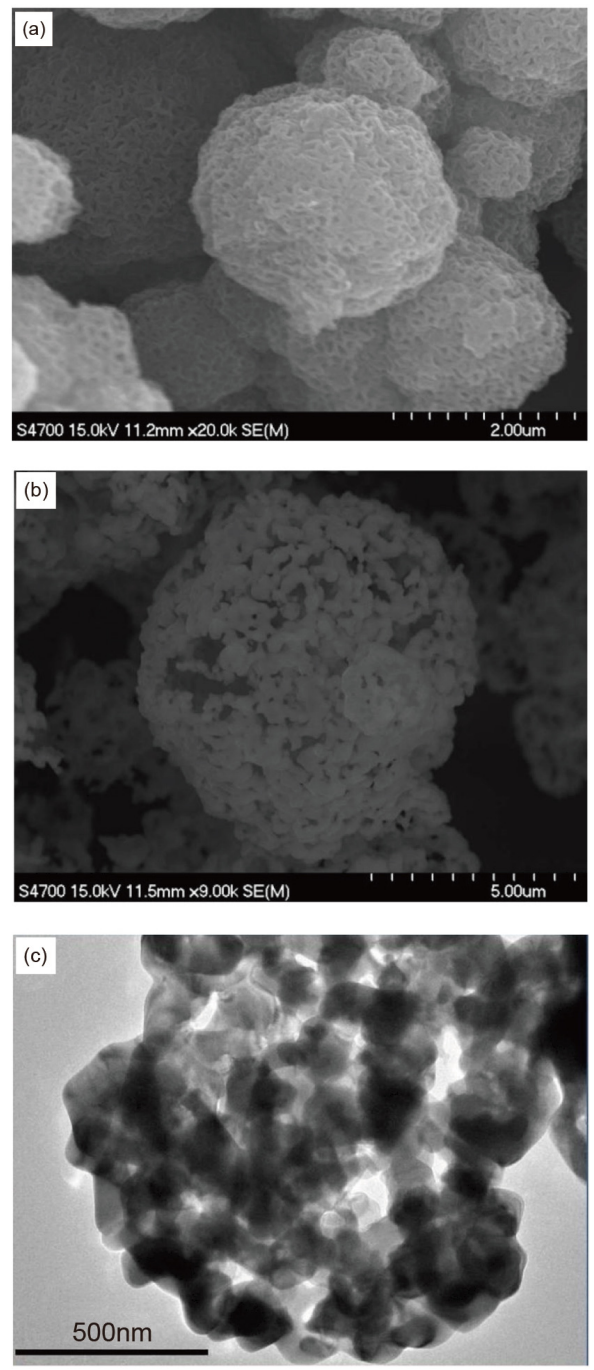

Fig. 10. (a, b) SEM images of porous iron oxide micro-particles fabricated by spray drying process. The calcination temperature was adjusted as (a) 500 and (b) $780{ }^{\circ} \mathrm{C}$. Scale bars indicate (a) 2 and (b) $5 \mu \mathrm{m}$. (c) TEM image of porous iron oxide micro-particles shown in (b). Scale bar is $500 \mathrm{~nm}$.

generated by the spray drying system were spherical shape. From the magnified microscope image displayed in Fig. 9(b), it can be seen that the surfaces of the polystyrene nanospheres in the composite particles are coated with a thin film, produced by the deposition of iron chloride during the self-organization process inside the drying chamber of the spray dryer.

Figure 10(a) contains a SEM image of the porous iron oxide micro-particles synthesized by the evaporation-driven self-assembly scheme outlined in Fig. 8. Calcination was performed at $500{ }^{\circ} \mathrm{C}$, which is a temperature high enough to remove the polymeric templates. The porous architectures of the iron oxide particles can be observed in this image, and it is evident that the macropores were successfully generated from the polystyrene nanosphere as sacrificial templates after high temperature calcination. When the calcination temperature was increased to $780{ }^{\circ} \mathrm{C}$, the morphologies of the porous iron oxide particles changed due to the high energy input during the heat treatment, as displayed in the SEM image in Fig. 10(b) and the TEM image in Fig. 10(c).

Porous iron oxide particles are important intermediate materials for the fabrication of porous metal powders, which can be applied in various industrial fields, including porous surface burners, artificial bones, thermal insulation, and sound absorbers [26-30].

After hydrogen reduction, the porous oxide particles shown in the SEM images in Fig. 10 can also be utilized to fabricate porous metal particles with potential importance in powder metallurgy industries. A study to investigate the use of the spray drying process and successive reduction step for the fabrication of porous metal powders is currently underway.

\section{CONCLUSIONS}

In this study, macroporous titania micro-particles were synthesized by a spray drying process using polymeric beads and titania nano-colloids as the template and precursor materials, respectively. The UV blocking properties and photocatalytic performance of the fabricated porous titania powders were measured for potential applications in cosmetics and water purification systems, respectively. When the macroporous titania powder was mixed with water, a reduction in the transmittance of light was observed in the 260 to $350 \mathrm{~nm}$ wavelength region, indicating that the porous materials can be used as UV blocking additives in sun creams. Rhodamine B dyes were decomposed when the macroporous titania particles were used as photocatalysts under UV light irradiation, implying that the porous micro-particles can be used to replace current commercial titania nano-colloids, but without skin absorption and toxicity. It was demonstrated that the proposed spray drying technique in this study can also be applied to fabricate porous iron oxide 
particles and further investigation is underway to utilize the particles to form porous metal powders after a reduction reaction.

\section{ACKNOWLEDGMENTS}

This research was supported by a grant (16CTAP-C114861-1) from Infrastructure and Transportation Technology Promotion Research Program funded by Ministry of Land, Infrastructure and Transport (MOLIT) of Korea Government and Korea Agency for Infrastructure Technology Advancement (KAIA).

\section{REFERENCES}

1. R. J. White, R. Luque, V. L. Budarin, J. H. Clark and D. J. Macquarrie, Chem. Soc. Rev. 38, 481 (2009).

2. J. H. Moon, G.-R. Yi, S.-M. Yang, D. J. Pine and S. B. Park, Adv. Mater. 16, 605 (2004).

3. K. Hieda, T. Hyodo, Y. Shimizu and M. Egahira, Sensors Actuat. B 133, 144 (2008).

4. X. J. Li, Q. M. Yu, J. H. Yang, Z. Z. Zeng and X. P. Jing, J. Electrochem. Soc. 154, H726 (2007).

5. C.-Y. Son, S.-B. Lee, S.-K. Lee, C. P. Kim and S. Lee, Korean J. Met. Mater. 48, 109 (2010).

6. S. Ratchapat, N. Viriya-empikul, K. Faungnawakij, T. Charinpanitkul and A. Soottitantawat, Sci. J. UBU 1, 40 (2010).

7. S. Zhang, L. Chen, S. Zhou, D. Zhao and L. Wu, Chem. Mater. 22, 3433 (2010).

8. Z. Wu and D. Zhao, Chem. Comm. 47, 3332 (2011).

9. L. Jiang, Y. Zhao and J. Zhai, Angew. Chem. Int. Ed. 43, 4338 (2004).

10. Q. Gao, J. Luo, X. Wang, C. Gao and M. Ge, Nanoscale Res. Lett. 10, 176 (2015).

11. T.-S. Kim, E.-W. Jeong, I.-K Choi, W. I. Park and Y.-R. Cho, Korean J. Met. Mater. 53, 294 (2015).

12. Y.-S. Cho, J. H. Moon, G.-R. Yi and S. M. Yang, J.
Disperse. Sci. Tech. 31, 368 (2010).

13. Y.-S. Cho and G.-R. Yi, J. Disperse. Sci. Tech. 31, 169 (2010).

14. Y.-S. Cho, T. Y. Kim, G.-R. Yi, Y. K. Kim and C. J. Choi, Bull. Kor. Chem. Soc. 33, 159 (2012).

15. S. Y. Lee, L. Gradon, S. Janeczko, F. Iskandar and K. Okuyama, ACS Nano 4, 4717 (2010).

16. F. Iskandar, M. Abdulla and K. Okuyama, Nano Lett 1, 231 (2001).

17. D. E. Geller, J. Weers and S. Heuerding, J. Aerosol Med. Pulm. Drug Deliv. 24, 175 (2011).

18. N. Tsapis, D. Bennett, B. Jackson, D. A. Weitz and D. A. Edwards, Proc. Natl. Acad. Sci. U.S.A. 99, 12001 (2002).

19. Y.-S. Cho, S. Y. Choi, Y. K. Kim and G.-R. Yi, J. Colloid Interf. Sci. 386, 88 (2012).

20. Y.-S. Cho, Y. K. Kim, S. H. Kim, D. C. Lim, J. G. Lee, Y. K. Baek and G.-R. Yi, Mater. Exp. 4, 91 (2014).

21. S.-M. Yang and O. O. Park, The Principles of the Flow of Micro-structured Fluids, 1st ed., pp. 17-18, Minumsa, Seoul (1997).

22. A. B. D. Nandiyanto, F. Iskandar and K. Okuyama, Chem. Eng. J., 152, 293 (2009).

23. C. S. Yah, G. S. Simate and S. E. Iyuke, Pak. J. Pharm. Sci., 25, 477 (2012).

24. Y.-S. Cho, G.-R. Yi, J.-M. Lim, S.-H. Kim, V. N. Manoharan, D. J. Pine and S.-M. Yang, J. Am. Chem,. Soc. 127, 15968 (2005).

25. I.-A. Oh, C. H. Shin and Y.-S. Cho, Korean J. Met. Mater. 54, 573 (2016).

26. S. A. Hashemi, M. Nikfar and R. Motaghedifard, Thermal Science 19, 11 (2015).

27. B. H. Smith, S. Szyniszewski, J. F. Hajjar, B. W. Schafer and S. R. Arwade, J. Constr. Steel Res. 71, 1 (2012).

28. K. M. Kulinowski, P. Jiang, H. Vaswani and V. L. Colvin, Adv. Mater. 12, 833 (2000).

29. Y.-H. Jo, Y.-H. Kim, Y. J. Jo, J. G. Seong, S. Y. Chang, P. J. Reucroft, S. B. Kim and W. H. Lee, Met. Mater. Int. 21, 337 (2015).

30. Y. J. Kim, J. W. Jang, D. W. Lee and S. Yi, Motoyama, Met. Mater. Int. 21, 673 (2015). 